\title{
Review of Medical Simulation Training for Endovascular Thrombectomy
}

\author{
Kieran Latham \\ Department of Civil Engineering \\ Liverpool John Moores University \\ Liverpool, United Kingdom \\ K.Latham@ljmu.ac.uk
}

Atif Waraich

Department of Computer Science

Liverpool John Moores University

Liverpool, United Kingdom

A.I.Waraich@ljmu.ac.uk

\author{
Patryk Kot \\ Department of Civil Engineering \\ Liverpool John Moores University \\ Liverpool, United Kingdom \\ P.Kot@ljmu.ac.uk \\ Mani Puthuran \\ Interventional Neuroradiologist \\ NHS Trust: The Walton Centre \\ Liverpool, United Kingdom \\ Mani.Puthuran@thewaltoncentre.nhs.uk
}

\author{
Dhiya Al-Jumeily \\ Faculty of Engineering and Technology \\ Liverpool John Moores University \\ Liverpool, United Kingdom \\ D.Aljumeily@ljmu.ac.uk \\ Arun Chandran \\ Interventional Neuroradiologist \\ NHS Trust: The Walton Centre \\ Liverpool, United Kingdom \\ Arun.Chandran@thewaltoncentre.nhs.uk
}

\begin{abstract}
This paper aims to discuss the state-of-the-art medical training simulators developed for the training and enhancement of technical skills related to complex procedures. The article discusses the skills and medical procedures reproduced by these simulators. Furthermore, the paper identifies the limitations of existing state-of-the-art technology and discusses the obstacles presented by these limitations. The paper then looks at novel approaches to these obstacles and systems currently being developed.
\end{abstract}

Keywords-Medical Engineering, Sensor Technology, Virtual Reality, Medical Training Simulation.

\section{INTRODUCTION}

This paper discusses the existing technologies available for medical simulation training for Endovascular Thrombectomy surgery for trainee clinicians. In the UK alone, there are over 100,000 people admitted into hospital following a stroke each year [1]. Out of all these cases, $85 \%$ are due to ischaemic stroke, which is where a cerebral infarction occurs due to the narrowing or blocking of arteries supplying blood to the brain [2]. At present, the number of clinicians qualified to perform Endovascular Thrombectomy on live patients is quite low. This predicament combined with it taking between 2-3 years of training before expert Interventional Neuroradiologists (INR) believe them to be capable of performing the procedure means that there needs to be an evaluation of existing methodologies in the training of this complicated procedure. Section II will discuss existing solutions to medical training simulation, the types of systems available, and how clinicians utilise them. Section III will investigate the limitations of existing medical training simulators identified in Section II. Section IV will investigate novel ways of solving the problems identified in Section III. Section V will discuss the current progress of the research project and the utilisation of different methods and technologies. Section VI will provide a discussion on the findings within this paper and what this research has identified. Section VII will discuss the opportunities for further investigation and novel applications. Section VIII will conclude the paper with a final summary of the paper.

\section{EXISTING MEDICAL TRAINING SimUlatorS}

As a response to ethical concerns growing from allowing trainees to operate on a patient under supervision from an experienced clinician, medical institutes have begun investing in the use of medical simulators, which can be used by trainees to receive surgical training and develop the technical skills required to perform operations successfully [3], [4].

\section{A. LapSIM Laparoscopic Training Simulator}

LapSIM is a screen-based training simulator with haptic feedback that is used to train clinicians in endoscopy. The platform allows trainees to develop their fine-motor and handeye coordination skills related to endoscopic procedures through a series of task modules and perform a virtual endoscopy [5].

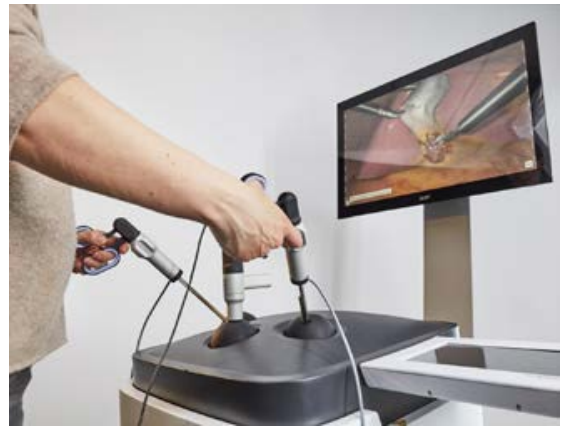

Fig 1. LapSIM Laparoscopic Training Simulator [19]

\section{B. Vascular Interventional Simulation Training (VIST) G5}

Mentice's Vascular Interventional Simulation Training (VIST) is a high-fidelity endovascular training simulator that allows trainee interventional neuroradiologists to conduct a full endovascular thrombectomy. The simulator breaks the procedure down into distinct phases; each one simulates a different step in the process of removing a blood clot inside a patient. The system uses real patient cases, which provides trainees with a model solution for them to complete [6].

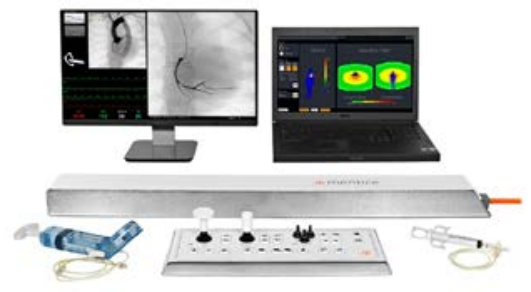

Fig 2. Mentice VIST G5 Endovascular Simulator [20] 


\section{Simbionix Arthro Mentor}

Simbionix's Arthro Mentor is a medical training simulator with haptic feedback that allows trainees to practise arthroscopic procedures and develop the relevant skills related to the anatomical structure of the shoulder and relevant biomechanics, and help build their knowledge of shoulder arthroscopy [7].

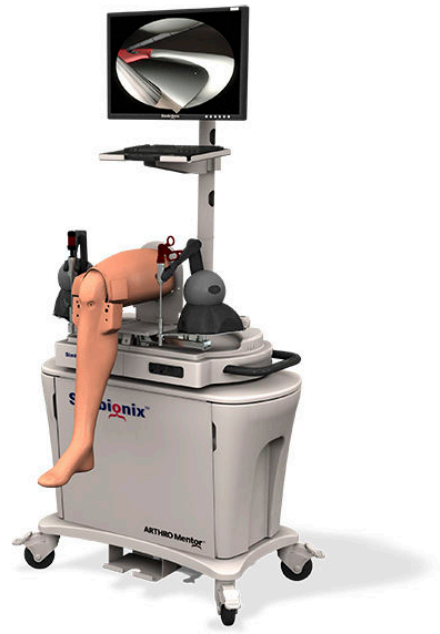

Fig 3. Simbionix Arthroscopic Simulator [8]

\section{LiMITATIONS OF EXISTING TECHNOLOGY}

\section{A. Lack of "on the job" training}

A serious obstacle to trainees in developing their technical skills and knowledge of complicated surgical procedures is the reduction in time available for them to access the operating room or operate on a patient to gain valuable real-life experience [5], [9], [10]. Regarding Mechanical Thrombectomy, this is a significant obstacle to overcome as one of the critical skills necessary to become proficient in this procedure is to guide a catheter and guidewire through the artery system effectively. Taking into account the physical pressure of the artery, how much force the trainee is applying, and the ability to ascertain whether they demonstrate controlled movement and are unlikely to cause damage [11].

\section{B. Lack of haptic feedback}

Fairhurst [9] identified in her work that there is a significant lack of realistic haptic and tactile feedback regarding Laparoscopic and Endoscopic procedures, citing this as an ongoing area of research. Meijden and Schijven [10] investigated the benefit of haptic and tactile feedback in virtual reality training in regards to Robot-Assisted Surgery (RAS), and Minimal Invasive Surgery (MIS) and identified that exposing training clinicians to haptic feedback early on improved their medical training performance. However, the paper indicated that a lack of parameters regarding accurate and effective haptic feedback exists and that the development of such a system would enhance training by combining existing visual feedback with haptic feedback to enhance performance. Pinzon, Byrns, and Zheng [12] identified that existing medical simulators lack haptic feedback due to the fact they were designed specifically to emulate tasks and medical equipment, as opposed to accurately simulating a

LJMU Faculty of Engineering and Technology and The Walton Centre NHS Foundation Trust medical procedure, and as such does not attempt to simulate this functionality.

\section{The learning curve}

Crossley [6] discusses in their work on performance metrics in mechanical thrombectomy (MT) the learning curve trainee doctors advance along during their training and the challenges and risks involved with the human interaction factors, which pose a risk to both patient and trainee. This article identifies that there are no established and reliable performance metrics related to the trainees learning curve. Gallagher [13] goes into more detail, describing the learning curve as a tool for trainers to understand how trainees are progressing, not just in terms of psychomotor skills, but also cognitively. The author also relates the learning curve to task automation, concerning procedure time and cases performed and believes that the goal should be for trainers to observe the trainees progress in developing these automatic skills before moving onto operating on a live patient.

\section{NOVEL APPROACHES TO IDENTIFIED ISSUES}

While the research discussed earlier highlights some exciting breakthroughs in this field, there are several limitations worth further investigation.

\section{A. Haptic feedback simulation}

As identified in the previous section, medical simulation trainers today lack haptic feedback of any sorts. In vascular intervention, in which this research project aims towards, the only two forms of feedback physicians have is visual feedback in the form of a monitor and haptic feedback by how the catheter and guidewire feel as they navigate the arteries. The limitation is that the existing platforms provide visual feedback. However, no system exists that provides haptic feedback to the user or any indication of how much force they are applying. Lack of physical feedback is a problem in that feel plays a huge part in the trainee's performance and is considered by expert doctors to be a key parameter in determining proficiency. By developing a sensor-based artery system, it is possible to simulate the arterial navigation, which is a crucial skill for trainees, and have them gain the experience and provide quantitative metrics to identify the amount of pressure applied and how forceful users are being.

\section{B. Quantifying the learning curve}

An issue surrounding Endovascular Thrombectomy training is the timeframe it takes to become considered proficient in the procedure, which is around 2-3 years long. A prominent theme across several papers is the learning curve trainees must advance along in order to become proficient. While some papers have looked at observing each completed task and observe the progress made with each completion. It is possible with machine learning algorithms to monitor several of these curves over time, with different levels of ability and create baselines to assess trainee progress compared to expert users, and better predict the timeframe.

\section{Quantitatively measuring user focus}

With advancements in technology, it is possible to observe and monitor physiological metrics regarding user focus and stress levels. The purpose of this would be to monitor trainees during a task and measure key parameters that would be identified through research and use it to identify 
their stress levels and correlate it with their learning curve to see where trainees are struggling or losing focus.

\section{METHODOLOGY}

This research will explore the development of the sensor system for pressure detection during arterial navigation; a prototype system was designed to measure the force applied to the sensor during arterial navigation. Sensors detect when a polyurethane tube is passing through another polyurethane tube to simulate a catheter moving through an artery. Furthermore, the initial development of a VRLE has been explored using photogrammetry and the $3 \mathrm{D}$ reproduction of a real-life environment to utilise in VR.

\section{A. Sensor System Development}

The initial step was to look at developing a system that could detect and measure the physical pressure of a catheter moving along an artery.

It involves connecting a Force Sensing Resistor (FSR) to an Arduino Nano. When the pressure gets applied to the pressure-sensitive layer, the conductive layers connect, and the resistance decreases proportionately to the amount of force applied. Additionally, the conductivity increases as physical forces are applied. This change is due to the fall in resistance.

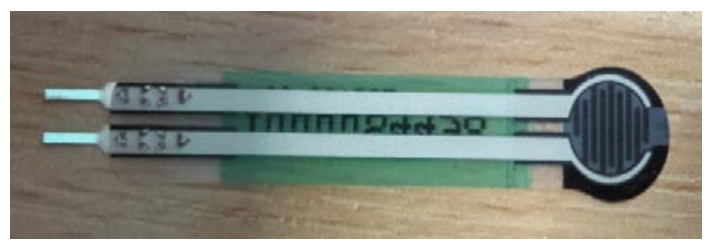

Fig 5. Force Sensing Resistor (FSR)

An Arduino Nano is used to communicate between the FSR and the PC, so that data can be accessed and recorded out into external software for different purposes, potentially so it can be accessed later and compared with updated datasets.

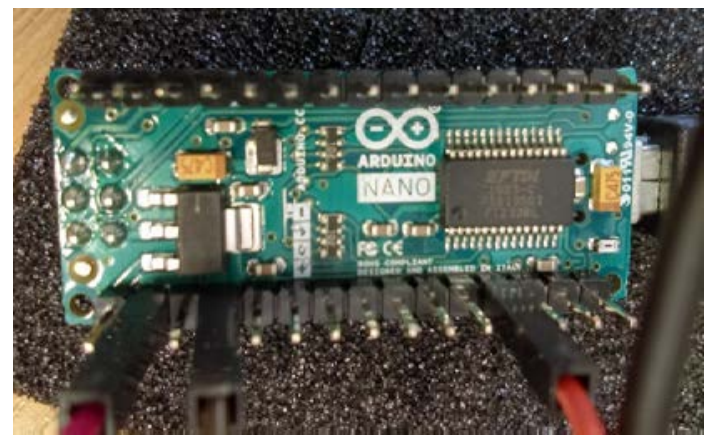

Fig 6. Arduino Nano Microprocessor

A program loaded onto the Arduino Nano reads for changes in the FSR and runs mathematical calculations to determine voltage and resistance, and force being read by the system. The program allows the data to be recorded for assessment later.

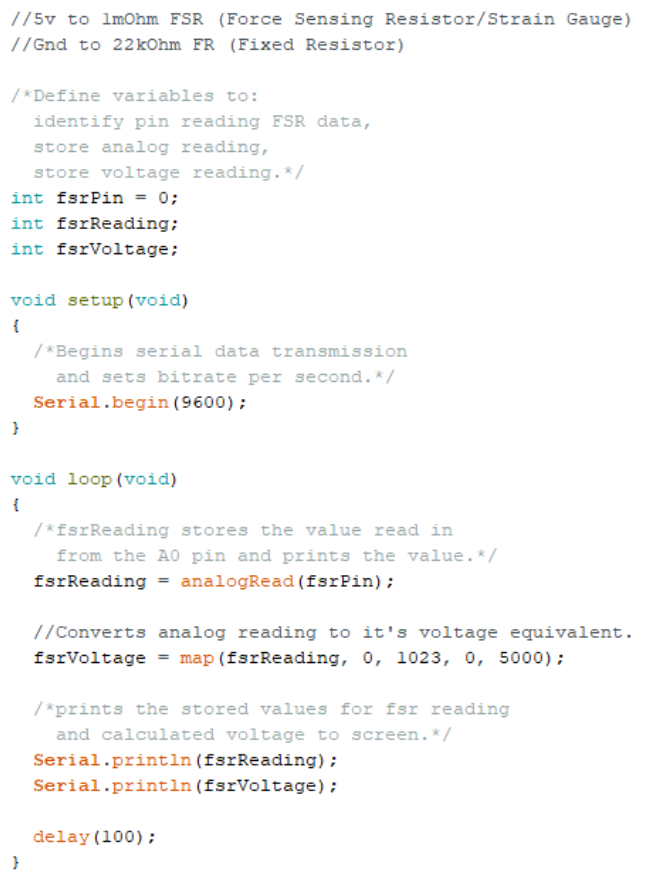

Fig 4. Code developed to measure FSR voltage

Initial tests utilised paper-based tubes of varying sizes to determine if sliding a smaller tube through a larger tube would trigger a reading from the sensor. These were considered successful and led to tests conducted with polyurethane tubing that provides flexibility and the ability to simulate the undulation and bending of arteries that provide trainees with resistance and challenge.

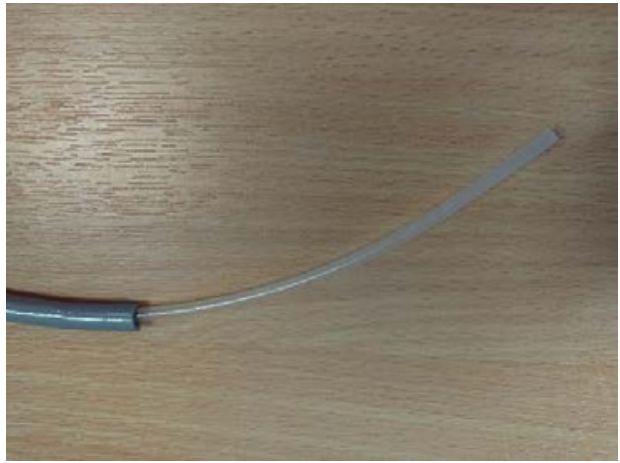

Fig 8. Polyurethane tubing simulating artery and catheter

Python code was developed to run a script that communicates with the Arduino Nano and prints out the data readings onto the screen for users to read. The script generates a timestamped Comma-Separated Value (CSV) file each time it is ran.

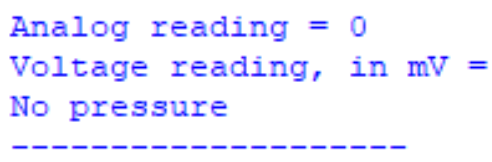

Fig 7. Python reading 'No pressure' from FSR 


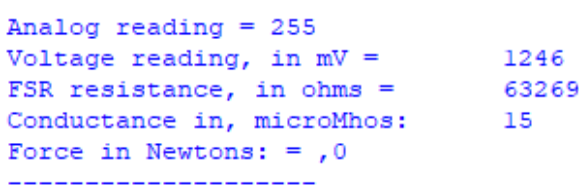

Fig 10. Python reading detected force from FSR

Testing the use of force-sensing resistors has shown that using a force-sensing resistor to measure physical pressure applied to a catheter or artery wall during arterial navigation is possible; however, further work is needed to produce precise results and identify the optimal values to measure when calculating force.

\section{B. Virtualisation and Immersion}

As a part of the development of a Virtual Reality Learning Environment, a live environment will be reconstructed in 3D to represent the working environment associated with the task conducted. In this case, an angioplasty theatre will be the environment visualised in virtual reality.

The virtualisation of a live working environment for medical training requires a $3 \mathrm{D}$ environment. The methods discussed outline how the environment gets produced and the output provided:

1) The first method looked at was photogrammetry, which converts key features in photographs to tie points, creating a point cloud in a 3D space and then generating a 3D mesh-based off of the point cloud, then exported as a 3D model. The benefit of photogrammetry is the creation of environments and models by way of image capture, which is mostly automated, except for userdefined variables to adjust the output of the application. The most significant limitation is that the mesh it generates has an excessive number of polygons and developed in 'tris' as opposed to quads, which are easier to work with and manipulate, the generated mesh during this study was $1,818,940$ polygons. Additionally, decimation causes noise and distortion to the mesh and textures and does not efficiently reduce the polycount.

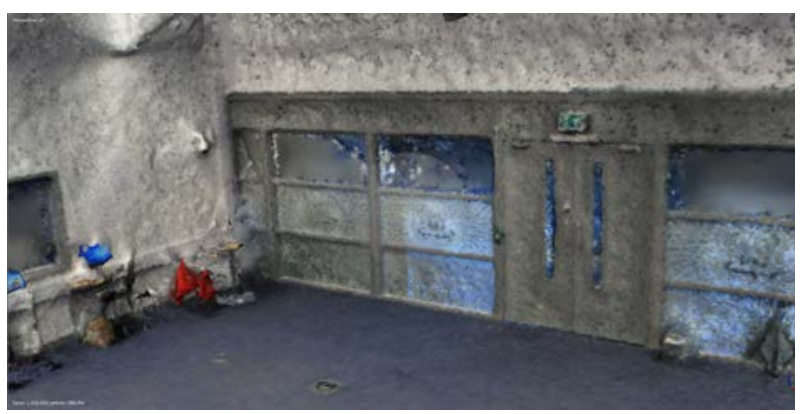

Fig 12. 3D world built from unstructured point cloud data

2) The second method investigated for the development of a $3 \mathrm{D}$ environment was to $3 \mathrm{D}$ model the environment by hand using reference images and measurement tools, such as existing point cloud data or measuring tape. The advantage of 3D modelling is that the user has full control over the design and development of the environment and can assess the geometry modelled in order to improve the efficiency of the final model. 3D modelling is more time consuming; however, as all the geometry has to be built manually and textured with UV maps before being usable by game engines [14]. Figure 11 demonstrates the use of 3D modelling for the level design of the Virtual Anthropology Museum. The total polycount for this environment is 239,580 polygons.

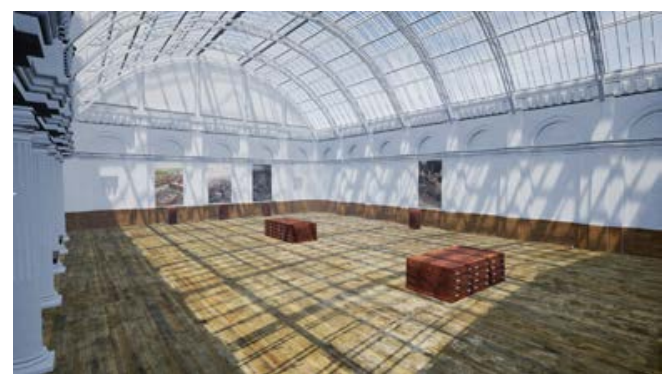

Fig 9. 3D world produced using image/scale references

3) The third method identified is inferred geometry. However, further work needs conducting and experimental work performed. Unlike photogrammetry that produces unstructured point clouds, this method uses the likes of FARO or LEICA point clouds to produce data that can be registered and structured in Autodesk software. Linking to AutoCAD allows users to extract geometrical features from the point cloud, such as edges, corners, planes, and cylinders, this can then be used to create a $3 \mathrm{D}$ environment from the point cloud, with a much more efficient result than photogrammetry with the whole scene in figure 12 being 852,348 [15].

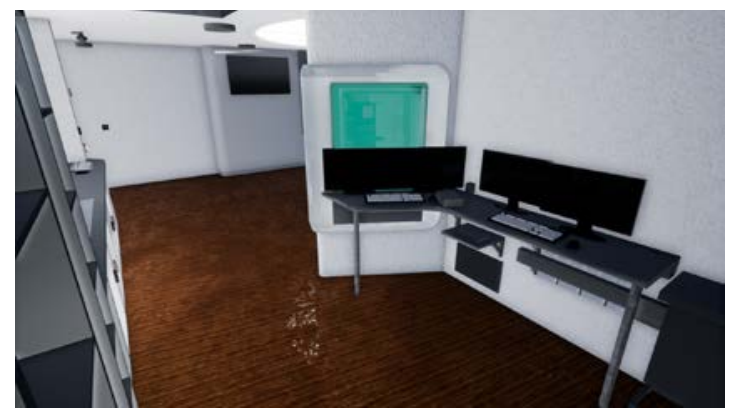

Fig 11. 3D world created with structured point cloud data [15]

The research investigated in 3D modelling for architectural visualisation and game development indicates that static meshes made for real-time rendering and virtual reality get sufficiently optimised. The optimisation involves tasks such as mesh decimation, which involves removing excessive or unnecessary geometry to reduce the polycount, opting instead to include specific details in texture files that can reproduce particular 3D or lighting effects.

\section{DISCUSSION}

The research conducted in this article demonstrates the variety of medical simulators available and the ability to simulate and train clinicians in a wide range of medical procedures. Based on the existing platforms, it is evident that most simulators are built as task simulations and are designed to allow training clinicians to develop their knowledge of the procedure, enhance their technical skills, and improve their 
knowledge of the human body. The applications highlighted in this article demonstrate the ability to improve medical training curriculum using simulation training. Medical Simulators provide training clinicians with a safe space to train within and associate themselves with the steps involved in the procedures and the risks involved [16]. They also provide supervising clinicians with a way of quantifying a trainee's performance and compare it to established parameters within the training program to determine the trainees level of performance in the procedure.

However, there are several critical limitations identified within these works that, if overcome, could allow for great strides to be made in enhancing the training of clinicians. Several articles established a link between the time it takes for a training clinician to become proficient in a procedure and the number of contact hours they receive with a patient. The link identified that the reduction of contact hours with patients meant training clinicians have to make up the training in simulation, which while training them in the procedure steps, fails to simulate certain aspects of surgery and inhibits the development of crucial skills.

Another obstacle to simulation training is the lack of haptic feedback provided by training platforms. As discussed earlier, a lot of medical training platforms are not built with haptics in mind and serve purely as task simulators for trainees to build a cognitive walkthrough of a procedure. Specific procedures, such as endovascular thrombectomy, requires clinicians to be able to feel the pressure they are applying to a catheter while navigating a patient's artery system. If doctors are unable to gain the ability to determine how much pressure they are applying to a catheter, they risk causing severe damage to a patient.

Furthermore, there are many references in research to the procedure's learning curve and the established differences between a beginning, novice, and expert surgeon [17]. Identifying the learning curve and level of ability can help indicate changes in performance [18]. Determining a practitioner's level of ability and readiness to perform a surgery is still decided by an expert user. While the learning curve is referred to in numerous articles, in theory, none seem to identify a means of utilising this learning curve in practice and using it to establish a practitioner's progress along their curve.

\section{FUTURE WORK}

From the limitations identified within this paper, there are numerous avenues for further investigation. One area of research could be the use of physiological parameters to observe a clinician's level of focus and attention to the task. An observation platform would allow experts to monitor the trainee during their practice and identify what parts of the procedure get performed autonomously and with little distraction or struggling. The observations could allow for more specific planning regarding training for the trainee by isolating parts of the procedure where they demonstrate loss of concentration or hesitation.

Currently, existing technology allows experts to observe trainees, but the decision on the progress remains a manual decision, improvements to the decision-making process are possible. The new approach will enable the decision to be backed up by the results from the simulation training platform to aid the decision of their competency. The system will provide experts with a way of continuously monitoring the trainee's progress and identify areas of weakness and how quickly they are achieving the expected levels of focus and autonomy to provide them with an estimated timeframe.

As identified earlier, the lack of feel and haptic feedback in simulators given to training clinicians reduces the overall quality of training provided. By developing a system that can recreate the feel of navigating a patient's arteries and feedback that pressure, training clinicians can develop the ability to feel the pressure applied during the real scenario surgery. This feeling can communicate back to the user, so they can improve their skills by identifying when they are applying excess force. Given how important this skill is to their training, it can provide supervising clinicians with a performance metric that can help to determine how efficient the trainee is at arterial navigation.

\section{VIII.CONCLUSION}

To summarise, there is extensive work being done to provide training and supervising clinicians with high-fidelity training platforms. The existing platforms provide training clinicians with the ability to develop a cognitive walkthrough of even the most complex procedures and the opportunity to develop a deeper understanding of the relevant anatomy and potential complications involved. Trainees can improve their confidence and develop their technical skills to an expert level through this form of training. Expert clinicians can use these platforms to observe the progress of less experienced clinicians without exposing them to the risks of performing surgery on a living patient. However, there are still multiple obstacles that, if addressed, will enhance the quality of the training provided for new clinicians in the future and improve their ability to transfer these skills from simulator to real-life scenario.

\section{REFERENCES}

[1] Stroke association, "State of the nation Stroke statistics," 2018

[2] Intercollegiate Stroke Working Party, "National clinical guideline for stroke Fifth Edition 2016," 2016.

[3] L. Li et al., "Application of virtual reality technology in clinical medicine.," Am. J. Transl. Res., vol. 9, no. 9, pp. 3867-3880, 2017.

[4] M. Maytin, T. P. Daily, and R. G. Carillo, "Virtual reality lead extraction as a method for training new physicians: A pilot study," PACE - Pacing Clin. Electrophysiol., vol. 38, no. 3, pp. 319-325, 2015.

[5] K. W. van Dongen, E. Tournoij, D. C. van der Zee, M. P. Schijven, and I. A. M. J. Broeders, "Construct validity of the LapSim: Can the LapSim virtual reality simulator distinguish between novices and experts?," Surg. Endosc., vol. 21, no. 8, pp. 1413-1417, Aug. 2007.

[6] R. Crossley et al., "Validation studies of virtual reality simulation performance metrics for mechanical thrombectomy in ischemic stroke," J. Neurointerv. Surg., pp. 1-7, 2019.

[7] P.-S. Marcheix, G. Vergnenegre, F. Dalmay, C. Mabit, and J.-L. Charissoux, "Learning the skills needed to perform shoulder arthroscopy by simulation," Orthop. Traumatol. Surg. Res., vol. 103, no. 4, pp. 483-488, Jun. 2017.

[8] Medical Expo, "Arthroscopy simulator / leg - ARTHRO Mentor ${ }^{\mathrm{TM}}$ - Simbionix - Videos," 2019. [Online]. Available: https://www.medicalexpo.com/prod/simbionix/product-81276629579.html. [Accessed: 21-Aug-2019].

[9] K. Fairhurst, A. Strickland, and G. Maddern, "The LapSim virtual reality simulator: Promising but not yet proven," Surgical Endoscopy, vol. 25, no. 2. Springer-Verlag, pp. 343-355, 08-Feb2011.

[10] O. A. J. van der Meijden and M. P. Schijven, "The value of haptic feedback in conventional and robot-assisted minimal invasive surgery and virtual reality training: a current review," Surg. Endosc., vol. 23, no. 6, pp. 1180-1190, Jun. 2009.

[11] I. Van Herzeele, R. Aggarwal, A. Choong, R. Brightwell, F. E. Vermassen, and N. J. Cheshire, "Virtual reality simulation 
objectively differentiates level of carotid stent experience in experienced interventionalists," J. Vasc. Surg., vol. 46, no. 5, pp. 855-863, 2007.

[12] D. Pinzon, S. Byrns, and B. Zheng, "Prevailing Trends in Haptic Feedback Simulation for Minimally Invasive Surgery," Surg. Innov., vol. 23, no. 4, pp. 415-421, Aug. 2016.

[13] A. G. Gallagher et al., "Virtual Reality Simulation for the Operating Room," Ann. Surg., vol. 241, no. 2, pp. 364-372, Feb. 2005.

[14] K. Latham et al., "A case study on the advantages of 3D walkthroughs over photo stitching techniques," Proc. - 2016 Int. Conf. Virtual Real. Vis. ICVRV 2016, no. 1, pp. 364-371, 2017.

[15] W. Hurst, K. Latham, D. O. Hare, A. Sands, S. O. Carroll, and R. J. Gandy, "A Case Study on the Development of a Virtual Reality Proton Beam Therapy Unit," Proc. - 2018 Int. Conf. Interact. Digit. Media, ICIDM 2018, pp. 1-13.

[16] Y. Munz, B. D. Kumar, K. Moorthy, S. Bann, and A. Darzi, "Laparoscopic virtual reality and box trainers: Is one superior to the other?," Surg. Endosc. Other Interv. Tech., vol. 18, no. 3, pp. 485-494, Mar. 2004.

[17] M. Paschold, T. Huber, S. Maedge, S. R. Zeissig, H. Lang, and W. Kneist, "Laparoscopic assistance by operating room nurses: Results of a virtual-reality study," Nurse Educ. Today, vol. 51, pp. 68-72, 2017.

[18] R. Aggarwal et al., "An evidence-based virtual reality training program for novice laparoscopic surgeons," Ann. Surg., vol. 244, no. 2, pp. 310-314, Aug. 2006.

[19] Surgical Science, "LapSim ${ }^{\circledR} 2018$ Annual Update | LapSim ${ }^{\circledR}$ | Surgical Science," 2018. [Online]. Available: https://surgicalscience.com/systems/lapsim/lapsim2018/.

[Accessed: 21-Aug-2019].

[20] Mentice, "VIST® G5 SIMULATOR," 2019. [Online]. Available: https://www.mentice.com/vist-g5. [Accessed: 21-Aug-2019]. 